\title{
A influência da Realidade Virtual como instrumento terapêutico para lombalgia em
}

\section{adultos jovens}

The influence of virtual reality as a therapeutic tool for low back pain in young adults

La influencia de la realidad virtual como herramienta terapéutica para la lumbalgia en adultos jóvenes

Beatriz Rithiely Henrique Ramos da Silva ORCID: https://orcid.org/0000-0003-3720-4448 Universidade Católica de Pernambuco, Brasil E-mail: beatriz.rithiely@ hotmail.com

Marina de Lima Neves Barros ORCID: https://orcid.org/0000-0002-3544-0538 Universidade Católica de Pernambuco, Brasil E-mail: marina.barros@unicap.br

Érica Patrícia Borba Lira Uchôa ORCID: https://orcid.org/0000-0003-4099-1876 Universidade Católica de Pernambuco, Brasil E-mail: erica.uchoa@unicap.br

Valéria Conceição Passos de Carvalho ORCID: https://orcid.org/0000-0001-8314-9000 Universidade Católica de Pernambuco, Brasil E-mail: valeria.passos@unicap.br

Maria Eduarda Siqueira Veras

ORCID: https://orcid.org/0000-0001-9400-2987 Universidade Católica de Pernambuco, Brasil E-mail: dudasveras1@gmail.com

Patrícia Lopes Ferreira de Lima ORCID: https://orcid.org/0000-0002-5464-5942 Universidade Católica de Pernambuco, Brasil E-mail: patricialopesflima @outlook.com

Ellen Raquel Lopes Rodrigues

ORCID: https://orcid.org/0000-0002-3375-0401 Universidade Católica de Pernambuco, Brasil E-mail: ellenrlopes5@gmail.com

\begin{abstract}
Resumo
Objetivo: Avaliar a utilização da Realidade Virtual como instrumento terapêutico para lombalgia em adultos jovens. Métodos: O estudo é de caráter quasi-experimental, de corte transversal descritivo e quantitativo, com a participação de 6 indivíduos, do sexo feminino, com idade entre 20 e 25 anos. Foi realizada avaliação postural através do Software para Avaliação Postural (SAPO) e a aplicação do Índice de Incapacidade de Oswestry (IIO) para avaliar as limitações e dificuldades laborais, antes e após as dez (10) sessões do Nintendo Wii. Assim como foi utilizada a Escala Visual Analógica (EVA) em todas as sessões antes e após a utilização do Nintendo Wii para avaliar a intensidade da dor. Resultados: As participantes obtiveram variação da dor lombar através da EVA, durante a avaliação, quatro (4) dos indivíduos relataram dor lombar moderada e após as 10 sessões a totalidade das voluntárias não apresentaram dor. E também, uma evolução satisfatória da capacidade funcional. Contudo, não houve mudanças posturais nos indivíduos participantes do estudo. Conclusão: A partir dos resultados encontrados, sugere-se que há indícios de melhora após a utilização da Realidade Virtual através do Nintendo Wii. Apesar disto, é recomendado que haja replicações deste estudo com uma amostra maior e mais diversificada para reforçar as evidências obtidas nesta pesquisa.
\end{abstract}

Palavras-chave: Lombalgia; Reabilitação; Realidade virtual.

\begin{abstract}
Objective: Evaluate to use of virtual reality as a tool therapeutic for low pain back in adults young. Methods: The study is almost experimental, descriptive, and quantitative cross-sectional, with the participation of 6 female subjects, aged between 20 and 25 years. The postural assessment was performed using the Software of Postural Analysis (SAPO) and the application of the Oswestry Disability Index (ODI) to assess work limitations and difficulties, before and after the ten (10) Nintendo Wii sessions. As well as the Visual Analogue Scale (VAS) was used in all sessions before and after using the Nintendo Wii to assess pain intensity. Results: The participants obtained variation in low
\end{abstract}


back pain through VAS, during the evaluation, four (4) of the individuals reported moderate low back pain and after the 10 sessions all volunteers did not pain present. And also, a satisfactory evolution of capacity functional. However, there were no changes postural in the study participants. Conclusion: From the results found, it is suggested that there are signs of improvement after using Virtual Reality through the Nintendo Wii. Despite this, it is recommended that there be replications of this study with a larger and more diverse sample to reinforce the evidence obtained in this research.

Keywords: Low back pain; Rehabilitation; Virtual reality.

\section{Resumen}

Objetivo: Evaluar el uso de la Realidad Virtual como herramienta terapéutica para el dolor lumbar en adultos jóvenes. Métodos: El estudio es casi experimental, transversal, descriptivo y cuantitativo, con la participación de 6 sujetos del sexo femenino, con edades comprendidas entre 20 y 25 años. La evaluación postural se realizó utilizando el Software de Evaluación Postural (SAPO) y la aplicación del Índice de Discapacidad de Oswestry (IDO) para evaluar las limitaciones y dificultades laborales, antes y después de las diez (10) sesiones de Nintendo Wii. Además de la Escala Visual Analógica (EVA) se utilizó en todas las sesiones antes y después de usar la Nintendo Wii para evaluar la intensidad del dolor. Resultados: Los participantes obtuvieron variación en el dolor lumbar mediante EVA, durante la evaluación, cuatro (4) de los individuos refirieron lumbalgia moderada y luego de 10 sesiones todos los voluntarios no presentaron dolor. Y también, una evolución satisfactoria de la capacidad funcional. Sin embargo, no hubo cambios posturales en las personas que participaron en el estudio. Conclusión: A partir de los resultados encontrados, se sugiere que existen señales de mejora luego de usar la Realidad Virtual a través de la Nintendo Wii. A pesar de ello, se recomienda que se realicen réplicas de este estudio con una muestra más amplia y diversa para reforzar la evidencia obtenida en esta investigación.

Palabras clave: Lumbalgia; Rehabilitación; Realidad virtual.

\section{Introdução}

A lombalgia, é a maior representante das síndromes dolorosas da coluna vertebral, depois da hipertensão arterial, é considerada a segunda doença crônica mais prevalente na população brasileira. Cerca de 27 milhões de pessoas, mais de 17\% da população acima dos 18 anos, têm o diagnóstico de patologias crônicas da coluna vertebral, representado pela lombalgia. (Hebert et al., 2017)

A origem da dor está localizada entre a $12^{\circ}$ vértebra torácica e prega glútea, devido à múltiplos fatores de risco, entre eles, o trabalho sentado por longas horas, atividades usuais como passar ou lavar roupas, sedentarismo, trabalhos que envolvam o levantamento repetitivo de objetos pesados, principalmente quando as cargas ultrapassam a força do trabalhador. (Santos, et al., 2019; Cargnin et al., 2019)

$\mathrm{O}$ tratamento da lombalgia é mais eficaz quando apresenta como foco, o paciente e não à sua lesão. Sendo assim, o tratamento dessa patologia precisa de um olhar biopsicossocial, dando importância a vários fatores, sendo eles: físicos, afetivos, comportamentais, sociais e cognitivos. (Thomas et al., 2016; Silva \& Martins, 2014)

Dentre as formas de tratar a lombalgia, a fisioterapia é considerada padrão principal para diminuição da sintomatologia, e apresenta vários recursos, como mobilizações, liberação miofascial, eletroterapia, acupuntura, alongamentos e exercícios. (Silva \& Martins, 2014).

Atualmente, com a avanço tecnológico, destaca-se a Realidade Virtual (RV) que recentemente ganha importância na visão dos profissionais de saúde. O potencial dessa tecnologia abrange a utilização de uma interação complexa entre paciente e máquina, e promove um feedback sensorial, cognitivo, psicológico e motor. (Jerald, 2015)

A RV é de extrema importância na preparação de um programa terapêutico que procure manter o interesse do paciente durante o tempo necessário para intervenção, e promove motivação através do seu caráter desafiador, garantindo o efeito almejado pela reabilitação. (Moraes et al., 2016)

Dessa forma, o Nintendo Wii que tem sido bastante utilizado na área da reabilitação, é um sistema simples do ponto de vista da aplicabilidade, com favorável relação custo-benefício. Especialmente o sistema Wii Fit que possibilita a eficiência do tratamento de disfunções motoras relacionadas ao equilíbrio, permitindo adaptações às limitações do paciente. (Aramaki et 
al., 2019)

Nessa perspectiva, os benefícios da RV utilizando o Nintendo Wii como ferramenta terapêutica na fisioterapia, incluem correções de postura e do equilíbrio, aumento da capacidade de locomoção, da força e da amplitude de movimento dos membros superiores e inferiores. Além da motivação do paciente, devido ao ambiente lúdico e atraente que, muitas vezes, não permite desistências, sendo alcançada mais rapidamente o propósito da terapia. (Vieira et al., 2014; Harvie et al., 2017; Soares, 2011)

Diante disso, é eminente as variadas estimulações oferecidas pela RV, tornando-se eficaz como uma nova ferramenta para a solução decorrentes de lesões musculoesqueléticas capaz de acarretar benefícios consideráveis. Portanto, esta pesquisa tem como objetivo avaliar a utilização da Realidade Virtual como instrumento terapêutico para lombalgia em adultos jovens.

\section{Metodologia}

O presente estudo está vinculado a Universidade Católica de Pernambuco, a Escola de Saúde e Ciências da Vida e ao curso de Fisioterapia. É parte integrante do projeto de pesquisa intitulado "Atenção Fisioterapêutica na Saúde do Indivíduo Adulto: Recursos de Avaliação, Perfil Epidemiológico e Intervenção”, cadastrado sob o No 442930-FTA-045-2019/3-7, financiada pela Fundação Antônio dos Santos Abrantes (FASA)/UNICAP e com aprovação do comitê de ética de pesquisa com seres humanos, da Unicap, sobre CAAE: 02861018.6.0000.5206 e parecer de número: 3.097.833.

A pesquisa é de caráter quasi-experimental, de corte transversal, descritivo e quantitativo. A escolha da amostra foi feita de forma intencional, de acordo com a quantidade de voluntários que aceitassem participar da pesquisa. A mesma foi desenvolvida nos laboratórios da Clínica Corpore Sano de Fisioterapia e Terapia Ocupacional, da Universidade Católica de Pernambuco.

Os participantes do estudo foram constituídos por adultos jovens de 20 a 25 anos, do sexo feminino, que apresentavam dor lombar. A amostra foi definida de forma intencional, de acordo com o quantitativo de participantes com lombalgia e disponíveis para participar da pesquisa, totalizando 6 indivíduos.

Foram utilizados os critérios de inclusão: Sexo feminino; apresentar dor lombar; idade entre 18 e 40 anos; assinar o Termo de Consentimento Livre e Esclarecido (TCLE). E como critérios de exclusão: apresentar alterações cognitivas; ter labirintite e epilepsia; apresentar disfunções neurológicas.

Na primeira fase do estudo, foi realizado um teste piloto com uma média de cinco participantes, com uma sessão para cada, com o intuito de avaliar qual o jogo será mais acessível e se enquadra aos objetivos da pesquisa.

Durante o estudo piloto, foram avaliadas todas as atividades do jogo Wii Fit - do Nintendo Wii, dentre elas, foram selecionadas as atividades de Yoga e Tonificação. No Yoga, foram escolhidas quatro posições, A Respiração Profunda, A média da Lua, o Guerreiro e a Árvore. Já na tonificação, os exercícios escolhidos foram, o equilíbrio sobre uma perna, Flexões e Torções em quatro apoios; por fim, Torções Laterais e Flexões Abdominais.

$\mathrm{Na}$ segunda fase do estudo, inicialmente, foi realizada uma palestra explicativa expondo as voluntárias, os pontos avaliados, como foram feitas as coletas das informações e apresentando potenciais benefícios e riscos deste projeto.

Após a palestra, foi perguntado as voluntárias se elas se dispunham, de forma livre e esclarecida, a participar do projeto. As que aceitaram participar, foi solicitada a assinatura do Termo de consentimento livre e esclarecido (TCLE).

Em seguida foi aplicado o questionário sócio-clínico-demográfico, a fim de traçar um perfil da amostra. Após o questionário foi realizada a verificação da dor lombar a partir da Escala Visual Analógica (EVA), que foi repetida todos os dias antes e após a utilização do Nintendo Wii. Para quantificar a incapacidade causada pela dor, foi utilizado o Índice de Incapacidade Oswestry (IIO) na avaliação e na reavaliação. E, por fim, foi feito um registro fotográfico na avaliação e na 
reavaliação baseado no protocolo do Software para Avaliação Postural (SAPO), onde foram avaliadas diversas medidas angulares do corpo humano remetendo à investigação de alterações posturais.

A EVA é uma escala graduada composta por números de 0 a 10 . A dor pode ser classificada como leve quando a escala for de 1 a 3; moderada, de 4 a 6 e forte, de 7 a 10. O número 0 na EVA representa a ausência de dor. (Carvalho, 2006).

Já o IIO, tem como objetivo detectar o grau de dor e de incapacidade dos indivíduos com lombalgia. A escala consiste em 10 seções com 6 itens que descrevem um grau crescente de incapacidade, sendo $0 \%$ a $20 \%$ o indicativo de incapacidade mínima, $21 \%$ a $40 \%$ incapacidade moderada e acima de $41 \%$ incapacidade intensa. A primeira seção avalia a intensidade da dor e as outras nove avaliam o efeito da dor lombar sobre as atividades diárias que podem ser interrompidas ou prejudicadas pela lombalgia. Cada seção possui A pontuação para determinar a interpretação dos resultados é a soma de pontos de cada seção marcada pelas voluntárias, divido por 50 e multiplicado por 100. (Masseli et al., 2007).

E, por fim, o protocolo SAPO é baseado na avalição do sujeito por quatro vistas fotográficas diferentes: anterior, posterior, lateral direita e lateral esquerda; gerando valores para o banco de dados e informando possíveis alterações. As referências anatômicas foram manualmente palpadas e demarcadas com bolas de isopor de quinze milímetros.

$\mathrm{Na}$ vista anterior foram marcados os pontos: glabela, trago D/E, mento, acrômio D/E, manúbrio do esterno, epicôndilo lateral D/E, cabeça do rádio $\mathrm{D} / \mathrm{E}$, cabeça da ulna $\mathrm{D} / \mathrm{E}$, espinha ilíaca ântero-superior $\mathrm{D} / \mathrm{E}$, trocânter maior do fêmur $\mathrm{D} / \mathrm{E}$, linha articular do joelho $\mathrm{D} / \mathrm{E}$, ponto medial da patela $\mathrm{D} / \mathrm{E}$, tuberosidade da tíbia $\mathrm{D} / \mathrm{E}$, maléolo lateral $\mathrm{D} / \mathrm{E}$, ponto entre a cabeça do $2^{\circ}$ e $3^{\circ}$ metatarso D/E.

$\mathrm{Na}$ vista posterior foram marcados os pontos: trago D/E, acrômio D/E, intersecção entre a margem medial e a espinha da escápula $\mathrm{D} / \mathrm{E}$, ângulo inferior da escápula $\mathrm{D} / \mathrm{E}$, espinha ilíaca póstero-superior $\mathrm{D} / \mathrm{E}$, epicôndilo lateral D/E, cabeça do rádio e a cabeça da ulna D/E, processo espinhoso de C7, T1, T3, T5, T7, T9, T11, T12, L1, L3, L4, L5 e S1, trocânter maior do fêmur $\mathrm{D} / \mathrm{E}$, linha articular do joelho $\mathrm{D} / \mathrm{E}$, ponto sobre a linha média da perna $\mathrm{D} / \mathrm{E}$, maléolo lateral $\mathrm{D} / \mathrm{E}$, ponto sobre o tendão do calcâneo D/E, maléolo medial D/E, calcâneo D/E.

$\mathrm{Na}$ vista lateral direita foram marcados os pontos: glabela, trago direito, mento, manúbrio do esterno, acrômio direito, epicôndilo lateral direito, ponto médio entre a cabeça do rádio e a cabeça da ulna direita, processo espinhoso de C7, T1, T3, T5, T7, T9, T11, T12, L1, L3, L4, L5 e S1, espinha ilíaca ântero-superior direita, espinha ilíaca póstero-superior direita, trocânter maior do fêmur direito, linha articular do joelho direito, ponto medial da patela direita, tuberosidade da tíbia, ponto sobre a linha média da perna direita, ponto sobre o tendão do calcâneo direito na altura média dos dois maléolos, calcâneo direito, maléolo lateral direito, ponto entre a cabeça do $2^{\circ}$ e $3^{\circ}$ metatarso direito.

E por fim, na vista lateral esquerda foram marcados os pontos: glabela, trago esquerdo, mento, manúbrio do esterno, acrômio esquerdo, epicôndilo lateral esquerdo, ponto médio entre a cabeça do rádio e a cabeça da ulna esquerda, processo espinhoso de C7, T1, T3, T5, T7, T9, T11, T12, L1, L3, L4, L5 e S1, espinha ilíaca ântero-superior esquerda, espinha ilíaca póstero-superior esquerda, trocânter maior do fêmur esquerdo, linha articular do joelho esquerdo, ponto medial da patela esquerda, tuberosidade da tíbia, ponto sobre a linha média da perna esquerda, ponto sobre o tendão do calcâneo esquerdo na altura média dos dois maléolos, calcâneo esquerdo, maléolo lateral esquerdo, e o ponto entre a cabeça do $2^{\circ}$ e $3^{\circ}$ metatarso esquerdo. (Ferreira el al., 2011; Duarte et al., 2005).

No terceiro momento, as participantes foram submetidas a dez (10) atendimentos de 20 minutos cada com o Nintendo Wii. Após cada sessão foi realizada uma reavaliação sobre intensidade e/ou melhora da dor lombar.

E, no final dos dez (10) atendimentos, foi realizada uma reavaliação com os mesmos critérios da avaliação inicial. Ao término da pesquisa de campo, as respostas serviram para alimentar uma planilha em Excel para análise descritiva e tabulação dos dados. 


\section{Resultados}

Fizeram parte dessa amostra 6 indivíduos, todos do sexo feminino, com média de idade 21,67 anos, \pm 2 . Quanto à lombalgia, a totalidade das mulheres relataram ter algum sintoma de dor lombar. Quanto a natureza da dor, 50\% (3) apresentavam dor na lombar e 50\% (3) dor na cervical, trapézio e lombar; já em relação a postura, 68\% (4) informaram dor na postura sentada e ereta. Assim como, 33\% (2) apresentavam histórico de trauma, histórico familiar de trauma, patologia pregressa e deformidade na coluna, conforme demonstra a Tabela 1.

Tabela 1. Questionário Sócio-clínico-demográfico (N=6).

\begin{tabular}{|c|c|c|c|c|c|}
\hline Variáveis & $\mathbf{N}(\%)$ & Média & DP & Min & Máx \\
\hline \multicolumn{6}{|l|}{ Sexo } \\
\hline Feminino & $6(100 \%)$ & - & - & - & - \\
\hline Idade (anos) & - & 21,67 & 2,0 & 1,9 & 21,6 \\
\hline \multicolumn{6}{|l|}{ Estado Civil } \\
\hline Solteiro(a) & $6(100 \%)$ & - & - & - & - \\
\hline \multicolumn{6}{|l|}{ Ocupação } \\
\hline Estudante & $6(100 \%)$ & - & - & - & - \\
\hline \multicolumn{6}{|l|}{ Histórico de trauma } \\
\hline Sim & $2(33 \%)$ & & & & \\
\hline Não & $4(67 \%)$ & & & & \\
\hline \multicolumn{6}{|c|}{ História familiar de problema na coluna } \\
\hline Sim & $2(33 \%)$ & & & & \\
\hline Não & $4(67 \%)$ & & & & \\
\hline \multicolumn{6}{|l|}{ Patologia pregressa } \\
\hline Sim & $2(33 \%)$ & & & & \\
\hline Não & $4(67 \%)$ & & & & \\
\hline \multicolumn{6}{|l|}{ Natureza da dor } \\
\hline Lombar & $3(50 \%)$ & & & & \\
\hline Cervical-trapézio-lombar & $3(50 \%)$ & & & & \\
\hline \multicolumn{6}{|l|}{ Postura da dor } \\
\hline Sentada & $1(16 \%)$ & & & & \\
\hline Ereta & $1(16 \%)$ & & & & \\
\hline Sentada e ereta & $4(68 \%)$ & & & & \\
\hline
\end{tabular}

Fonte: Autores.

A Tabela 2, demostra as características da ocorrência da dor conforme a Escala Visual Analógica (EVA) e sua classificação, e é possível observar que 83\% das mulheres apresentaram dores moderadas na lombar durante a avaliação, antes de serem submetidas a primeira sessão com o Nintendo Wii. 
Tabela 2. Ocorrência da dor conforme a classificação pela EVA - Antes do Nintendo Wii.

\begin{tabular}{lc}
\hline Variável & Total $(\mathrm{n}=6)$ \\
\hline Sem dor & 1 \\
Dor leve & 1 \\
Dor moderada & 4 \\
Dor intensa & 0 \\
\hline
\end{tabular}

Fonte: Autores.

Segundo a Tabela 2, uma (1) voluntária não apresentou dor, uma (1) apresentou dor leve, quatro (4) apresentaram dor moderada, e nenhuma relatou dor.

A Tabela 3, demostra a ocorrência da dor conforme a Escala Visual Analógica (EVA), após 20 minutos da primeira sessão utilizando o Nintendo Wii, e é possível observar que 33\% das mulheres não apresentaram dor, 50\% dor leve e $17 \%$ dor moderada.

Tabela 3. Ocorrência da dor conforme a classificação pela EVA - Após o uso Nintendo Wii.

\begin{tabular}{lc}
\hline Variável & Total $(\mathrm{n}=6)$ \\
\hline Sem dor & 2 \\
Dor leve & 3 \\
Dor moderada & 1 \\
Dor intensa & 0 \\
\hline
\end{tabular}

Fonte: Autores.

De acordo com a Tabela 3, duas (2) voluntárias não apresentaram dor, três (3) apresentaram dor leve, uma (1) apresentou dor moderada, e nenhuma relatou dor.

A Tabela 4, demostra a ocorrência da dor lombar após os 10 atendimentos, no início da décima sessão antes de ser utilizado o Nintendo Wii. Demonstrando que $16 \%$ não apresentaram dor, $68 \%$ dor leve e $16 \%$ dor moderada.

Tabela 4. Ocorrência da dor conforme a classificação pela EVA - Antes do Nintendo Wii.

\begin{tabular}{lc}
\hline Variável & Total $(\mathrm{n}=6)$ \\
\hline Sem dor & 1 \\
Dor leve & 4 \\
Dor moderada & 1 \\
Dor intensa & 0 \\
\hline
\end{tabular}

Fonte: Autores.

Segundo a Tabela 4, uma (1) voluntária não apresentaram dor, quatro (4) apresentaram dor leve, uma (1) apresentou dor moderada, e nenhuma relatou dor. 
Já a Tabela 5, demostra a ocorrência da dor lombar no final da décima sessão depois de ser utilizado o Nintendo Wii. Podendo-se observar que a $100 \%$ das mulheres não apresentaram dores.

Tabela 5. Ocorrência da dor conforme a classificação pela EVA - Após o uso do Nintendo Wii.

\begin{tabular}{lc}
\hline Variável & Total $(\mathrm{n}=6)$ \\
\hline Sem dor & 6 \\
Dor leve & 0 \\
Dor moderada & 0 \\
Dor intensa & 0 \\
\hline
\end{tabular}

Fonte: Autores.

De acordo com a Tabela 5, seis (6) voluntárias não relataram dor.

Em relação ao Índice de Incapacidade de Oswestry (IIO), a pontuação total foi obtida somando o número de pontos de cada seção marcadas pelas voluntárias, divido por 50 e multiplicado por 100. Na Tabela 6 e 7, encontra-se a interpretação dos resultados através do IIO.

Tabela 6. Interpretação dos resultados através do IIO - Avaliação.

\begin{tabular}{lc}
\hline Variável & Total $(\mathrm{n}=6)$ \\
\hline Incapacidade mínima & 3 \\
Incapacidade moderada & 3 \\
Incapacidade intensa & 0 \\
\hline
\end{tabular}

Fonte: Autores.

De acordo com a Tabela 6 , três mulheres (50\%) durante a avaliação no primeiro atendimento, foram classificadas a partir da sua pontuação com incapacidade moderada, e três (50\%) incapacidade mínima.

Tabela 7. Interpretação dos resultados através do IIO - Reavaliação.

\begin{tabular}{lc}
\hline Variável & Total $(\mathrm{n}=6)$ \\
\hline Incapacidade mínima & 5 \\
Incapacidade moderada & 1 \\
Incapacidade intensa & 0 \\
\hline
\end{tabular}

Fonte: Autores.

Conforme a Tabela 7, após os 10 atendimentos, na reavaliação, foi repetido o questionário e 5 voluntárias (83\%) foram classificadas com incapacidade mínima e apenas uma (17\%) apresentou incapacidade moderada.

Na Tabela 8, é possível observar os resultados da avaliação postural antes das 10 sessões utilizando o Nintendo Wii, por meio da vista anterior através do Software para Avaliação Postural (SAPO), e pode-se verificar que todas as mulheres apresentaram algum tipo de alteração postural. 
Tabela 8. Valores medidos em ângulos através do SAPO - Vista Anterior.

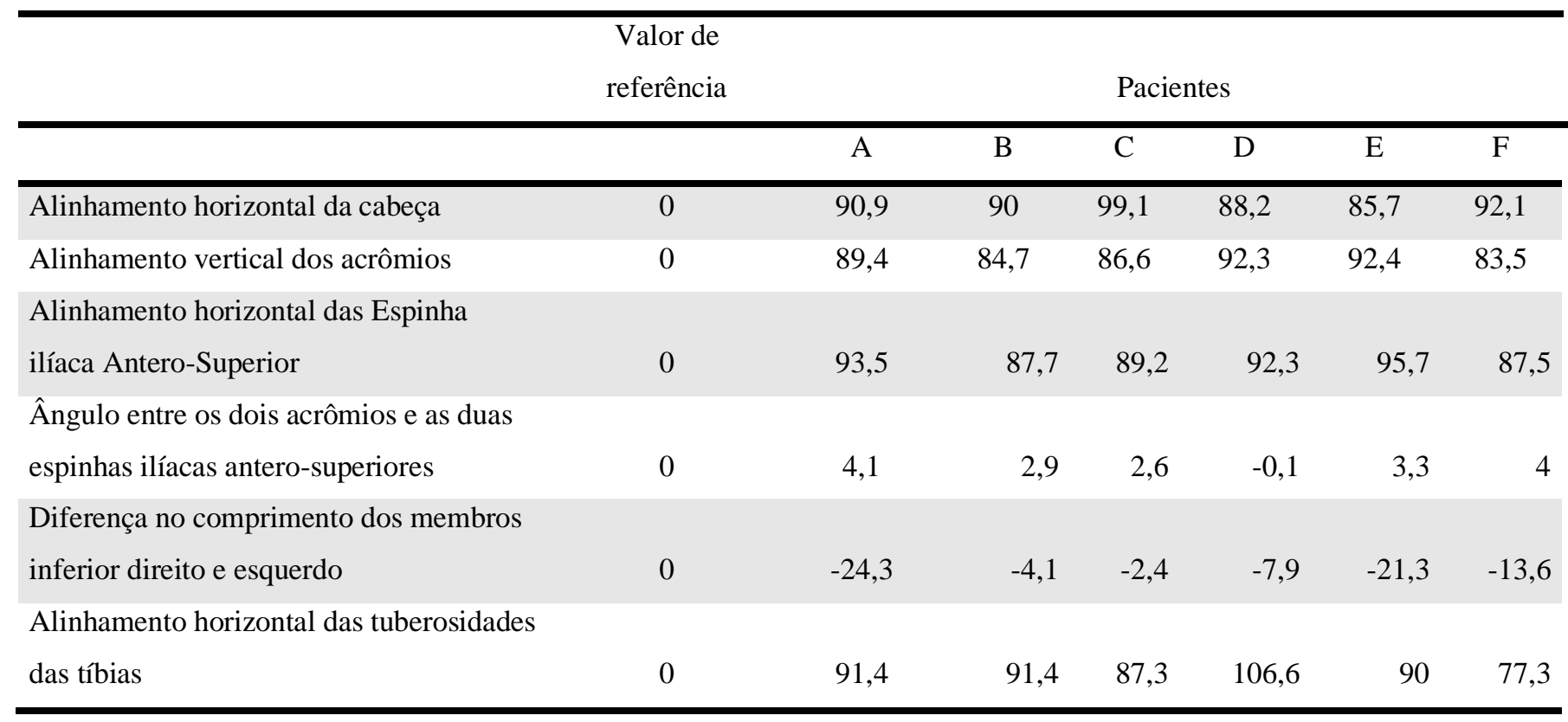

Fonte: Autores.

De acordo com a Tabela 8, as voluntárias A, B, C, D, E e F obtiveram o ângulo do alinhamento horizontal da cabeça positivo, ou seja, anti-horário, o que significa que a cabeça está rodada para o lado direito. Em relação ao alinhamento horizontal dos acrômios foi possível identificar que todos os pacientes apresentaram valores positivos, resultando no acrômio esquerdo mais elevado.

Se tratando do Alinhamento horizontal das espinhas ilíacas ântero-superiores (EIAS) todas as voluntárias possuíam valores positivos, ou seja, EIAS esquerda está mais alta que a direita. Já o angulo entre os dois acrômios e as duas EIAS os pacientes A, B, C, E e F tiveram valores positivos, sendo assim, a distância entre o acrômio e a EIAS direita é menor no lado esquerdo, devido a inclinação para a direita; e o paciente D apresentou ângulos negativos, indicando que a distância entre o acrômio e a EIAS direita é maior no lado esquerdo, indicando inclinação para a esquerda. Já a o resultado da diferença no comprimento dos membros inferiores direito e esquerdo (MID-E) foram valores negativos, assim todos os pacientes apresentaram o membro inferior direito (MID) maior que o membro inferior esquerdo (MIE). E por fim, no alinhamento horizontal das tuberosidades das tíbias todos os pacientes apresentaram valores positivos, ou seja, altura das tuberosidades da tíbia está menor do lado direito.

Na Tabela 9, é possível observar os resultados encontrados na avaliação postural antes das 10 sessões utilizando o Nintendo Wii, por meio da vista posterior.

Tabela 9. Valores medidos em ângulos através do SAPO - Vista Posterior.

\begin{tabular}{|c|c|c|c|c|c|c|c|}
\hline & $\begin{array}{l}\text { Valor de } \\
\text { referência }\end{array}$ & \multicolumn{6}{|c|}{ Pacientes } \\
\hline & & A & B & $\mathrm{C}$ & $\mathrm{D}$ & $\mathrm{E}$ & $\mathrm{F}$ \\
\hline $\begin{array}{l}\text { Assimetria horizontal da escápula em } \\
\text { relação à T3 }\end{array}$ & 0 & 2 & 0 & 1,9 & 20,5 & 9,6 & 0 \\
\hline
\end{tabular}


Conforme a Tabela 9, a assimetria horizontal da escápula em relação à T3 as mulheres A, C, D e E apresentaram valores positivos indicando que a escápula direita está mais elevada que a esquerda, e as voluntárias B e F, apresentaram ângulo 0 não possuindo assimetrias.

$\mathrm{Na}$ Tabela 10, é possível observar os resultados encontrados na avaliação postural antes das 10 sessões utilizando o Nintendo Wii, por meio da vista lateral direita.

Tabela 10. Valores medidos em ângulos através do SAPO - Vista Lateral Direita.

\begin{tabular}{lcccccccc}
\hline & $\begin{array}{c}\text { Valor de } \\
\text { referência }\end{array}$ & & & & \multicolumn{2}{c}{ Pacientes } \\
& & & & A & B & C & E & F \\
\hline Alinhamento horizontal da cabeça (C7) & 0 & 124,2 & 126,2 & 127,8 & 138,6 & 129,2 & 140,1 \\
Alinhamento vertical da cabeça (acrômio) & 0 & 77 & 70,4 & 60,7 & 73,7 & 67,4 & 70,5 \\
Alinhamento vertical do tronco & 0 & -86 & -89 & $-93,4$ & $-89,8$ & $-89,4$ & $-88,6$ \\
Alinhamento horizontal da pelve & 0 & $-69,7$ & $-80,7$ & 59,8 & 59,9 & 68,3 & 56,5 \\
\hline
\end{tabular}

Fonte: Autores.

De acordo com a Tabela 10, todas as voluntárias apresentaram o alinhamento horizontal da cabeça em relação a C7 positivo, e o alinhamento vertical da cabeça em relação ao acrômio, também demonstrou ângulos positivos constituindo anteriorização da cabeça. Já o alinhamento vertical do tronco, há uma possível diminuição ou retificação da cifose torácica nas voluntárias porque os ângulos foram negativos. Já o alinhamento horizontal da pelve, as voluntárias A e B tiveram valores negativos e com isso, sugere aumento da lordose lombar, e as mulheres C, D, E e F obtiveram ângulos positivos, apresentando diminuição da lordose lombar.

Na Tabela 11, é possível observar os resultados encontrados na avaliação postural antes das 10 sessões utilizando o Nintendo Wii, por meio da vista lateral esquerda.

Tabela 11. Valores medidos em ângulos através do SAPO - Vista Lateral Esquerda.

\begin{tabular}{|c|c|c|c|c|c|c|c|}
\hline & $\begin{array}{l}\text { Valor de } \\
\text { referência }\end{array}$ & \multicolumn{6}{|c|}{ Pacientes } \\
\hline & & $\mathrm{A}$ & $\mathrm{B}$ & $\mathrm{C}$ & $\mathrm{D}$ & $\mathrm{E}$ & $\mathrm{F}$ \\
\hline Alinhamento horizontal da cabeça (C7) & 0 & 48,1 & 41 & 50,6 & 38,5 & 50,6 & 40 \\
\hline $\begin{array}{l}\text { Alinhamento vertical da cabeça } \\
\text { (acrômio) }\end{array}$ & 0 & 101,5 & 102,6 & 112,6 & 116,2 & 117,7 & 98,6 \\
\hline Alinhamento vertical do tronco & 0 & $-87,1$ & $-89,3$ & -89 & $-84,7$ & $-85,1$ & $-90,7$ \\
\hline Alinhamento horizontal da pelve & 0 & $-95,9$ & $-99,8$ & 119,1 & 113,2 & 110,6 & 115,9 \\
\hline
\end{tabular}

Fonte: Autores.

Conforme a Tabela 11, quanto aos resultados do alinhamento horizontal da cabeça em relação a C7 e o alinhamento vertical da cabeça, em relação ao acrômio todos os ângulos foram positivos, ou seja, todas as mulheres apresentavam anteriorização da cabeça. Em relação ao alinhamento horizontal do tronco, há uma possível diminuição ou retificação da cifose torácica nas voluntárias porque os ângulos foram negativos. Já no alinhamento horizontal da pelve, as voluntarias A e B, 
tiveram valores negativos e com isso, sugere aumento da lordose lombar, e as mulheres C, D, E e F obtiveram ângulos positivos, apresentando diminuição da lordose lombar.

Na Tabela 12, é possível observar os resultados da avaliação postural na reavaliação após as 10 sessões utilizando o Nintendo Wii, por meio da vista anterior através do Software para Avaliação Postural (SAPO), e é possível observar que todas as voluntárias permaneceram com alguma alteração postural.

Tabela 12. Valores medidos em ângulos através do SAPO - após as 10 sessões - Vista Anterior.

\begin{tabular}{|c|c|c|c|c|c|c|c|}
\hline & Valor de referência & & & Pacie & & & \\
\hline & & A & B & $\mathrm{C}$ & $\mathrm{D}$ & $\mathrm{E}$ & $\mathrm{F}$ \\
\hline Alinhamento horizontal da cabeça & 0 & 90 & 92 & 95 & 90,9 & 91 & 85,1 \\
\hline Alinhamento horizontal dos acrômios & 0 & 92,8 & 94,2 & 88,2 & 91,5 & $-92,2$ & 84,3 \\
\hline Alinhamento horizontal das EIAS & 0 & 88,4 & 89,4 & 90 & 86,8 & $-87,6$ & 86 \\
\hline $\begin{array}{l}\text { Ângulo entre os dois acrômios e as } \\
\text { duas espinhas ilíacas ântero-superior }\end{array}$ & 0 & $-4,4$ & $-4,8$ & 1,8 & $-4,7$ & 4,3 & 1,8 \\
\hline $\begin{array}{l}\text { Diferença no comprimento dos } \\
\text { membros inferiores direito e esquerdo }\end{array}$ & 0 & 4,5 & 2,4 & 4,1 & $-0,7$ & 14,4 & 10,1 \\
\hline $\begin{array}{l}\text { Alinhamento horizontal das } \\
\text { tuberosidades das tíbias }\end{array}$ & 0 & 82 & 90 & 94,2 & 94 & 86,1 & 95,1 \\
\hline
\end{tabular}

Fonte: Autores.

De acordo com a Tabela 12, as participantes A, B, C, D, E e F continuaram apresentando o ângulo do alinhamento horizontal da cabeça positivo, ou seja, anti-horário, o que significa que a cabeça está rodada para o lado direito. Em relação ao alinhamento horizontal dos acrômios, as mulheres A, B, C, D e F permaneceram com valores positivos, resultando no acrômio esquerdo mais elevado, e a mulher E, apresentou valor negativo, constituindo o acrômio direito mais elevado.

Se tratando do Alinhamento horizontal das espinhas ilíacas ântero-superior (EIAS) as pacientes A, B, C, D e F continuaram com valores positivos, ou seja, EIAS esquerda está mais alta que a direita, já a paciente E, apresentou valor negativo constituindo a EIAS direita mais alta. Já o ângulo entre os dois acrômios e as duas EIAS das voluntárias A, B e D apresentaram valores negativos, contudo a distância entre o acrômio e EIAS direita é maior do que no lado esquerdo, indicando inclinação para a esquerda; e as mulheres C, E e F continuaram com os valores positivos, sendo assim, a distância entre o acrômio e a EIAS direita é menor no lado esquerdo, indicando inclinação para a direita. Em relação a diferença no comprimento dos membros inferiores direito e esquerdo MI(D-E) as voluntárias A, B, C, E e F apresentaram valores positivos indicando o MID menor que o MIE, e a mulher D apresentou valores negativos constituindo o MID maior que o MIE. E por fim, no alinhamento horizontal das tuberosidades das tíbias todas as voluntarias continuaram apresentando valores positivos, ou seja, altura das tuberosidades das tíbias está menor do lado direito.

Na Tabela 13, é possível observar os resultados encontrados na avaliação postural após as 10 sessões utilizando o Nintendo Wii, por meio da vista posterior. 
Tabela 13. Valores medidos em ângulos através do SAPO - após as 10 sessões - Vista Posterior.

\begin{tabular}{lccccccc}
\hline & Valor de referência & & \multicolumn{3}{c}{ Pacientes } \\
\hline & & $\mathrm{A}$ & $\mathrm{B}$ & $\mathrm{C}$ & $\mathrm{D}$ & $\mathrm{E}$ & $\mathrm{F}$ \\
\hline $\begin{array}{l}\text { Assimetria horizontal da escápula em } \\
\text { relação à T3 }\end{array}$ & 0 & 6,3 & 9,8 & 10,7 & 8,5 & 8,1 & 3,0 \\
\hline
\end{tabular}

Fonte: Autores.

Conforme a Tabela 13, a relação a assimetria horizontal da escápula em relação à T3 todas as voluntárias apresentaram valores positivos indicando que a escápula direita está mais elevada que a esquerda.

Na Tabela 14, é possível observar os resultados encontrados na avaliação postural após as 10 sessões utilizando o Nintendo Wii, por meio da vista lateral direita.

Tabela 14. Valores medidos em ângulos através do SAPO - após as 10 sessões - Vista Lateral Direita.

\begin{tabular}{lccccccc}
\hline & Valor de referência & \multicolumn{7}{c}{ Pacientes } \\
\hline & & A & B & C & D & E & F \\
\hline Alinhamento horizontal da cabeça (C7) & 0 & 123,4 & 131,2 & 121,8 & 139,9 & 126,7 & 132 \\
$\begin{array}{l}\text { Alinhamento vertical da cabeça } \\
\text { (acrômio) }\end{array}$ & 0 & 122,8 & 120,8 & 117,7 & 67,3 & 66,2 & 61,4 \\
Alinhamento vertical do tronco & 0 & $-85,6$ & $-87,2$ & $-87,1$ & $-94,3$ & $-91,1$ & -86 \\
Alinhamento horizontal da pelve & 0 & $-68,5$ & $-63,7$ & $-66,6$ & 70,8 & 68 & 73,3 \\
\hline
\end{tabular}

Fonte: Autores.

De acordo a Tabela 14, todas as voluntárias permaneceram com os ângulos do alinhamento horizontal da cabeça em relação a C7 positivos, e o alinhamento vertical da cabeça com relação ao acrômio, também demonstrou ângulos positivos constituindo anteriorização. Em relação ao alinhamento vertical do tronco, os resultados permaneceram negativos, sugere-se uma diminuição ou retificação da cifose torácica. Já no alinhamento horizontal da pelve sugere hiperlordose lombar porque as voluntarias A, B e C obtiveram valores negativos. E por fim, as mulheres D, E e F continuaram com os ângulos positivos e com isso, sugere uma retificação da lordose lombar.

E por fim, a Tabela 15 é possível observar os resultados encontrados na avaliação postural após as 10 sessões utilizando o Nintendo Wii, por meio da vista lateral esquerda. 
Tabela 15. Valores medidos em ângulos através do SAPO - após as 10 sessões - Vista lateral esquerda.

\begin{tabular}{|c|c|c|c|c|c|c|c|}
\hline & \multirow[t]{2}{*}{ Valor de referência } & \multicolumn{6}{|c|}{ Pacientes } \\
\hline & & A & $\mathrm{B}$ & $\mathrm{C}$ & $\mathrm{D}$ & $\mathrm{E}$ & $\mathrm{F}$ \\
\hline $\begin{array}{l}\text { Alinhamento horizontal da cabeça } \\
\text { (C7) }\end{array}$ & 0 & 51,8 & 48,1 & 45,8 & $-50,6$ & -42 & $-35,7$ \\
\hline $\begin{array}{l}\text { Alinhamento vertical da cabeça } \\
\text { (acrômio) }\end{array}$ & 0 & 62,8 & 68,3 & 62,3 & 114 & 107,9 & 108,4 \\
\hline Alinhamento vertical do tronco & 0 & $-91,5$ & $-89,3$ & $-96,8$ & $-88,5$ & $-89,6$ & $-88,7$ \\
\hline Alinhamento horizontal da pelve & 0 & $-113,3$ & $-117,9$ & -116 & 109 & 106,7 & 99,1 \\
\hline
\end{tabular}

Fonte: Autores.

Conforme a Tabela 15, os resultados do alinhamento horizontal da cabeça em relação a C7 e o alinhamento vertical da cabeça, em relação ao acrômio foram todos ângulos positivos, ou seja, todas as mulheres apresentavam anteriorização da cabeça. Em relação ao alinhamento horizontal do tronco, há uma possível diminuição ou retificação da cifose torácica nas voluntárias porque os ângulos permaneceram negativos. Já no alinhamento horizontal da pélvis, as voluntarias A, B e C, tiveram ângulos negativos e com isso, sugere aumento da lordose lombar, e as mulheres D, E e F obtiveram ângulos positivos, apresentando diminuição da lordose lombar.

\section{Discussão}

Thomas et al. (2016) e Yelvar et al. (2017), afirmam que o tratamento da lombalgia precisa ser biopsicossocial, dando importância a fatores físicos, sociais, afetivos e comportamentais e é preciso dar importância para o paciente como um todo e não se deter somente a sua lesão. Portanto, a Realidade Virtual (RV) traz a possibilidade de um envolvimento global do indivíduo pois fornece um feedback imediato e se torna uma maneira lúdica de tratamento. (Park \& Yim, 2016).

Um estudo realizado por Kim et. al. (2014) com 30 pacientes do sexo feminino de meia idade que apresentaram dor lombar crônica, divididas em grupo experimental $(n=15)$ e controle $(n=15)$, com duração de 12 sessões, ao longo de quatro semanas, em média por 30 minutos, revelou diferenças significativas entre o escore da Escala Visual Analógica (EVA) pré e pós utilização do Yoga no Wii Fit. O grupo experimental apresentou uma redução significativa na EVA de 7,00 ( \pm 0,89) pontos para 2,27 $( \pm 1,10)$ pontos, e o grupo controle mostrou uma diminuição significativa na EVA de 6,95 $( \pm 0,79)$ pontos para 4,63 $( \pm 1,91)$ pontos. Corroborando com os resultados do presente estudo, no qual foi realizado 10 sessões durante 4 semanas, por aproximadamente 20 minutos, e os participantes também apresentaram reduções importantes da EVA, sendo que no primeiro dia antes da utilização do Nintendo Wii Fit, $83 \%$ das mulheres apresentaram dor moderada, e na $10^{\mathrm{a}}$ sessão, $100 \%$ das mulheres não apresentaram dor na lombar.

Já na avaliação do presente estudo, através do Índice de Incapacidade de Oswestry (IIO) foi observado que 50\% das mulheres apresentaram incapacidade mínima e 50\% incapacidade moderada; na reavaliação após as 10 sessões, $83 \%$ apresentaram incapacidade mínima e 17\% incapacidade moderada. Esse achado é semelhante também ao estudo de Kim et. al. 
(2014), nas quais as pacientes tiveram melhoras no IIO de 34,91 $( \pm 6,19)$ pontos para $13,82( \pm 7,65)$ pontos, no final da utilização do Nintendo Wii Fit.

De acordo com Itakussu et al. (2015), há diversos benefícios do Nintendo Wii na reabilitação, como as correções posturais, treino de equilíbrio, aumento da capacidade de locomoção e da amplitude de movimento, além da motivação de fazer algo diferente. Por isso, as respostas fisiológicas do Nintendo Wii já foram estudadas para reabilitação cardiopulmonar, no combate a obesidade, em algumas mialgias agudas como a tendinite e no controle postural. (Cruz \& Menuchi et al., 2015).

Em relação a fotogrametria foi utilizado o Software para Avaliação Postural (SAPO) que é um recurso com o objetivo de avaliar o alinhamento e controle postural. Também pode ser importante para registrar as mudanças posturais com o passar do tempo, propiciando maior precisão do que outras formas de mensuração. (Souza, 2011). Dentre as diversas alterações encontradas, as principais foram anteriorização da cabeça, retificação da cifose torácica, retificação da lordose lombar e hiperlordose lombar. Na avaliação e após as 10 sessões foi feita a análise postural, e as voluntárias do presente estudo não apresentaram melhoras nas alterações posturais.

Esse achado, corrobora com o estudo de Almeida et al. 2019, que apresentou considerável número de mulheres com alguma alteração da curvatura fisiológica, sendo $82 \%$ com hiperlordose lombar, $18 \%$ com retificação lombar e $38 \%$ com anterioridade da cabeça.

De acordo com Wiederhold (2014), o tratamento baseado na exposição a realidade virtual gera distrações que pode aliviar os sintomas de dor nos pacientes. Esse achado corrobora com os resultados dos estudos de Yelvar et al. (2017) e Kim et. al. (2014), que demostra reduções significativas na dor e cinesiofobia após o uso da realidade virtual.

Assim como Park et al. (2013), em um estudo feito com trabalhadores de uma fábrica de pneus, utilizaram a eletrotermofototerapia em conjunto com o Nintendo Wii, e obtiveram resultados significantes em relação a limitações emocionais, fadiga, energia e redução da dor lombar.

\section{Conclusão}

A partir dos resultados encontrados, sugere-se que há indícios de melhora após a utilização da Realidade Virtual através do Nintendo Wii, em mulheres que apresentavam lombalgia, apresentando resultados satisfatórios na redução da dor e na melhora da capacidade funcional. Apesar disto, o estudo possuiu algumas limitações, como a quantidade de indivíduos avaliados, a falta de um grupo controle, e os poucos estudos existentes envolvendo pessoas com essas características.

Desta forma, para a realização de trabalhos futuros, é recomendado que haja replicações deste estudo com a inserção de um grupo controle, amostra maior de pacientes com variadas idades e aumento na quantidade de sessões, para reforçar as evidências obtidas nesta pesquisa.

\section{Referências}

Almeida, A. O. P., Nogueira, E. R., Sousa, J. C. M., Vieira, A. G., Assis, E. V., \& Dias, M. J. (2019). Alterações posturais e principais sintomatologias em pacientes com afecções de coluna vertebral. Journal of Medicine and Health Promotion. 4(3):1170-1179. http://jmhp.fiponline.edu.br/pdf/cliente=13$8 f 16 \mathrm{~b} 619$ faec $24 \mathrm{~b} 87809 \mathrm{c} 4 \mathrm{~b} 845 \mathrm{~d} 27258$.pdf

Aramaki, A. L., Sampaio, R. F., Reis, A. C. S., Cavalcanti, A., \& Dutra, F. C. M. S. (2019). Virtual reality in the rehabilitation of patients with stroke: an integrative review. Arquivos de Neuro-Psiquiatria, 77(4):268-278. https://doi .org/10 .1590/0004-282x20190025

Cargnin, Z. A., Schneider, D. G., Vargas, M. A. O., \& Schneider, I. J. C. (2019). Atividades de trabalho e lombalgia crônica em especial em trabalhadores de enfermagem. Acta Paulista de Enfermagem, Dez; 32(6): 707-713. https://doi .org/10 .1590/1982-0194201900097

Carvalho, D. S., \& Kowacs, P. A. (2006). Avaliação da intensidade de dor. Migrâneas cefaleias. Out/Dez;9 (4):164-168. https://headachemedicine. com. br/assessment-of-pain-intensity/

Cruz, R. V. S., D’Alencar, M. S., \& Menuchi, M. R. T. P. (2015). A utilização do Nintendo Wii como ferramenta terapêutica em programas de reabilitação traumato-ortopédica. Revista Pesquisa em Fisioterapia. Out;5(2):153-16 .http://dx .doi .org/10 .17267/2238-2704rpf .v5i2 .596 
Duarte, M., Ferreira, E. A., Maldonado, E. P., \& Freitas, A. Z. (2005). Documentação sobre o SAPO - Software para avaliação postural. http://pesquisa .ufabc .edu .br/bmclab/sapo/

Ferreira, E. A., Duarte, M., Maldonado E. P., Bersanetti A. A., \& Marques, A. P. (2011). Quantitative assessment of postural alignment in young adults based on photographs of anterior, posterior, and lateral views. Journal of manipulative and physiological therapeutics. 34(6):371-380. http://pesquisa.ufabc.edu .br/bmclab/pubs/jmpt11.pdf

Harvie, D. S., Smith, R. T., Hunter, E. V., Davis, M. G., Sterling, M., \& Moseley, G. L. (2017). Using visuo-kinetic virtual reality to induce illusory spinal movement: The MoOVi Illusion. PeerJ Journals. 5, e3023:1-16. https://doi .org/10 .7717/peerj .3023

Hebert, S. K., Filho, T. E. P. B., Xavier, R., \& Jr, A. G. P. (2017) Ortopedia e Traumatologia: Princípios e Prática. São Paulo: Artmed.

Itakussu, E. Y., Valenciano, P. J., Trelha, C. S., \& Marchiori, L. L. M. (2015). Benefícios do treinamento de exercícios com o Nintendo Wii na população de idosos saudáveis: uma revisão de literatura. Revista CEFAC. Maio/Jun;17(3):936-944. https://www.scielo.br/pdf/rcefac/v17n3/1982-0216-rcefac-17-03-00936 .pdf

Thomas, J. S., France, C. R., Applegate, M. E., Leitkam, S. T., \& Walkowski, S. (2016). Feasibility and Safety of a Virtual Reality Dodgeball Intervention for Chronic Low Back Pain: A Randomized Clinical Trial. The Journal of Pain. 17(12):1302-1317.https://doi .org/10 .1016/j.jpain.2016.08.011

Jerald, J. (2015). The VR book: human-centered design for virtual reality. Califórnia: Morgan \& Claypool Publishers.

Park, J., Lee, S., \& Ko, D. (2013). The Effects of the Nintendo Wii Exercise Program on Chronic Work-related Low Back Pain in Industrial Workers. Journal of Physical Therapy Science. 25(8):985-988. https://doi .org/10 .1589/jpts .25 .985

Kim, S., Min, W., Kim, J., \& Lee, B. (2014). The Effects of VR-based Wii Fit Yoga on Physical Function in Middle-aged Female LBP Patients. Journal of Physical Therapy Science. 26(4):549-552. https://doi.org/10.1589/jpts.26 .549

Masseli, M. R., Fregonesi, C. E. P. T., Faria, C. R. S., Bezerra, M. I. S., Junges, D., \& Nishioka, T. H. (2007). Índice Funcional de Oswestry após cirurgia para descompressão de raízes nervosas. Fisioterapia em Movimento. 20(1):115-122. https://periodicos.pucpr.br/index.php/fisio/article/view/18853

Moraes, V. B., Andrade, M. M. A., Toyoda, C. Y. \& Araujo, R. C. T. (2016). O uso do videogame Nintendo Wii como recurso terapêutico para idosos: uma análise da atividade na perspectiva da Terapia Ocupacional. Cadernos Brasileiros de Terapia Ocupacional. 24(4):705-714. http://dx.doi.org/10 .4322/01044931.ctoAO0640

Park, J., \& Yim, J. E. (2016). A new approach to improve cognition, muscle strength, and postural balance in community-dwelling elderly with a 3-D virtual reality Kayak program. Tohoku Jounal Of Experimental Medicine. 238(1):1-8. https://doi.org/10.1620/tjem.238 .1

Santos, M. A., Lunkes, L. C., Ribeiro, A. O. S., \& Souza, A. C. (2019). Dor lombar e fatores de risco na terceira infância. Fisioterapia em Movimento. 32, e003210: 1-9. https://dx .doi.org/10.1590/1980-5918.032.ao10

Silva, A. N., \& Martins, M. R. I. (2014). Dor, cinesiofobia e qualidade de vida de pacientes com dor lombar. Revista Dor. 15(2):117-120. https://doi .org/10 $.5935 / 1806-0013.20140023$

Soares, F. A. C. (2011). Influência da prática virtual de Yoga sobre o controle postural de mulheres idosas utilizando o Nintendo Wii. Dissertação (Mestrado em Bioengenharia) - Bioengenharia, Universidade de São Paulo: São Carlos. https://doi.org/10.11606/D.82.2011.tde-17072012-102920

Souza, J. A., Pasinato, F., Basso, D., Corrêa, E. C. R., \& Silva, A. M. T. (2011). Biofotogrametria confiabilidade das medidas do protocolo do software para avaliação postural (SAPO). Revista Braileira Cineantropometria e Desempenho Humano. Ago;13(4):299-305. https://doi.org/10.1590/1980-0037 $.2011 v 13 n 4 p 299$

Vieira, G. P., Araújo, D. F. G. H., Leite, M. A. A., Orsini, M., \& Correa, C. L. (2014) Realidade virtual na reabilitação física de pacientes com doença de Parkinson. Jounal Of Human Growth and Development. 24(1):31-41. http://pepsic .bvsalud .org/scielo .php?script=sci_arttext\&pid=S010412822014000100005\&lng=pt\&tlng=pt

Wiederhold, B. K., \& Bouchard, S. (2014). Advances in Virtual Reality and Anxiety Disorders. Springer.

Yelvar, G. D. Y., Çırak, Y., Dalkılınç, M., Demir, Y. P., Guner, Z., \& Boydak, A. (2017). Is physiotherapy integrated virtual walking effective on pain, function, and kinesiophobia in patients with non-specific low-back pain? Randomised controlled trial. European Spine Jounal. 26(2): 538-545. https://doi .org/10.1007/s00586-016-4892-7. 